\title{
Solutions of Large Integral-Equation Problems with Preconditioned MLFMA
}

\author{
Özgür Ergül ${ }^{1}$, Tahir Malas ${ }^{1}$, Alper Ünal ${ }^{1}$, and Levent Gürel ${ }^{1,2}$ \\ ${ }^{1}$ Department of Electrical and Electronics Engineering \\ ${ }^{2}$ Computational Electromagnetics Research Center (BiLCEM) \\ Bilkent University, TR-06800, Bilkent, Ankara, Turkey \\ lgurel@ee.bilkent.edu.tr
}

\begin{abstract}
We report the solution of the largest integralequation problems in computational electromagnetics. We consider matrix equations obtained from the discretization of the integral-equation formulations that are solved iteratively by employing parallel multilevel fast multipole algorithm (MLFMA). With the efficient parallelization of MLFMA, scattering and radiation problems with millions of unknowns are easily solved on relatively inexpensive computational platforms. For the iterative solutions of the matrix equations, we are able to obtain accelerated convergence even for ill-conditioned matrix equations using advanced preconditioning schemes, such as nested preconditioners based on an approximate MLFMA. By orchestrating these diverse activities, we have been able to solve a closed geometry formulated with the CFIE containing 33 millions of unknowns and an open geometry formulated with the EFIE containing 12 millions of unknowns, which are the largest problems of their classes, to the best of our knowledge.
\end{abstract}

Index Terms - Electromagnetic scattering, surface integral equations, iterative methods, multilevel fast multipole algorithm, parallelization, preconditioning techniques, metamaterials.

\section{INTRODUCTION}

For the numerical solutions of the scattering and radiation problems in electromagnetics, integral-equation formulations provide accurate results when they are discretized appropriately by using small elements with respect to wavelength. For perfectly conducting geometries, combined-field integral equation (CFIE) is commonly used for closed surfaces. For open surfaces, however, electric-field integral equation (EFIE) is used to properly formulate the problems. With the simultaneous discretization of the scatterer and the integral equations, dense matrix equations are obtained, where the solutions can be performed iteratively using efficient acceleration methods such as the multilevel fast multipole algorithm (MLFMA) [1]. On the other hand, accurate solutions of many real-life problems require discretizations with millions of elements leading to matrix equation with millions of unknowns. We consider the solutions of these large problems by employing a parallel MLFMA on a cluster of relatively inexpensive processors connected via special fast networks. Using robust preconditioners including a nested preconditioner based on an approximate MLFMA (AMLFMA), iterative solutions are performed efficiently, even for ill-conditioned matrix equation that are obtained from EFIE.

\section{MLFMA SOLUTIONS OF INTEGRAL EQUATIONS}

For the solutions of the scattering and radiation problems involving three-dimensional conducting surfaces, discretization of EFIE or CFIE leads to $N \times N$ dense matrix equation

$$
\sum_{n=1}^{N} Z_{m n}^{E, C} a_{n}=v_{m}^{E, C}, \quad m=1, \ldots, N
$$

where $Z_{m n}^{E, C}$ represents the matrix element, i.e., interaction of $m$ th testing and $n$th basis functions, $v_{m}^{E, C}$ represents the elements of the excitation vector obtained by testing the incident fields, and $a_{n}$ for $n=1,2, . ., N$ are the unknown coefficients. We apply a Galerkin scheme and choose the testing and basis functions as Rao-Wilton-Glisson (RWG) functions [2]. Matrix equations in (1) are solved iteratively, where the matrix-vector multiplications are accelerated by MLFMA [1] as

$$
\overline{\boldsymbol{Z}}^{E, C} \cdot \boldsymbol{x}=\overline{\boldsymbol{Z}}_{N F}^{E, C} \cdot \boldsymbol{x}+\overline{\boldsymbol{Z}}_{F F}^{E, C} \cdot \boldsymbol{x} .
$$

In (2), only the near-field interactions denoted by $\overline{\boldsymbol{Z}}_{N F}^{E, C}$ are calculated directly and stored in the memory, while the farfield interactions are computed approximately in a groupby-group manner. Based on the factorization of the Green's functions, aggregation, translation, and disaggregation steps are performed in a multilevel scheme. This way, the overall complexity of the matrix-vector multiplications is reduced to $\mathcal{O}(N \log N)$ for an $N \times N$ dense matrix equation.

\section{Solutions of Closed Surfaces by CFIE}

Formulations of closed geometries can be performed by employing CFIE, which is free of the internal-resonance problem and produces well-conditioned matrix equations. As an example, we consider the solution of a scattering problem involving a sphere of radius $96 \lambda$ illuminated by a plane wave. The discretization of the problem with a mesh size of $\lambda / 10$ leads to $33,791,232$ unknowns. This is the largest integralequation problem ever solved in computational electromagnetics, to the best of our knowledge. Iterative solution of the problem is achieved by a biconjugate-gradient-stabilized (BiCGS) algorithm accelerated by a parallel MLFMA. The solution is performed on a cluster of quad-core Intel Xeon 5355 processors connected via an Infiniband network. Parallelizing the solution into 16 process, setup of the program takes 177 


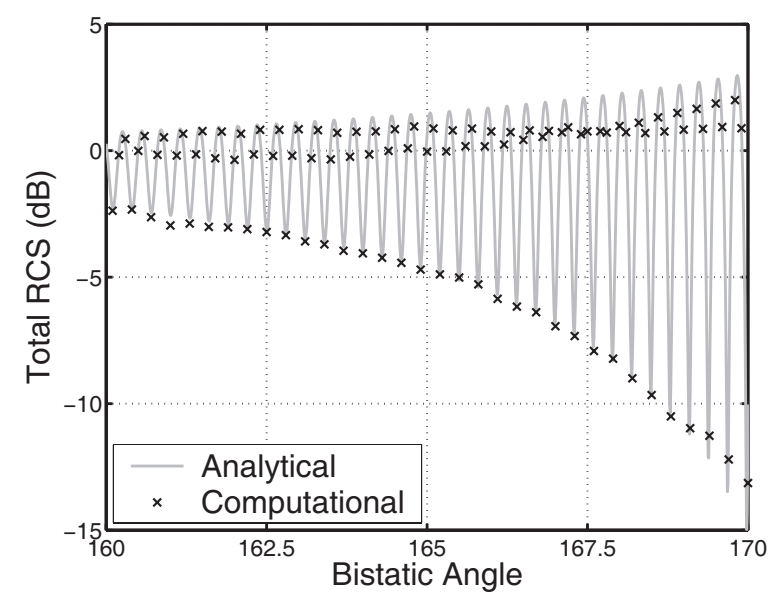

(a)

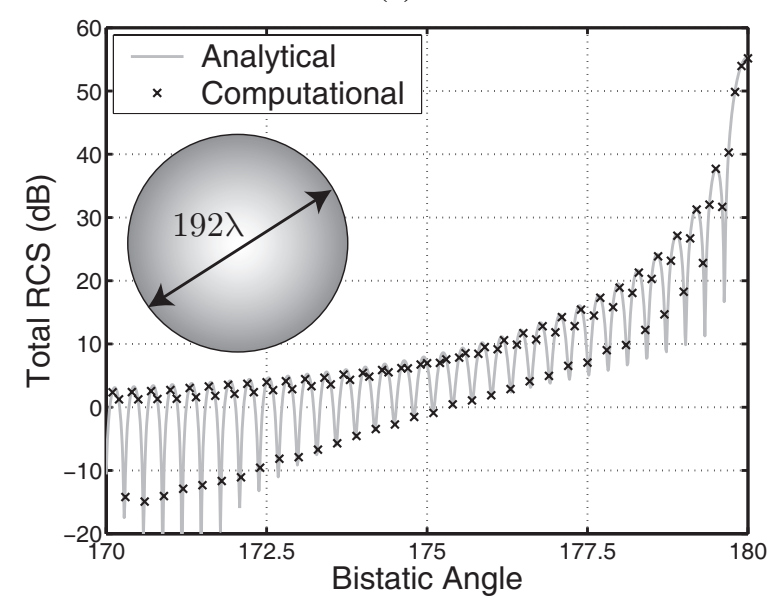

(b)

Fig. 1. Bistatic RCS of a sphere of radius $96 \lambda$ in the (a) $160^{\circ}-170^{\circ}$ and (b) $170^{\circ}-180^{\circ}$ ranges, where $180^{\circ}$ corresponds to the forward-scattering direction. Computational values obtain by the solution of a 33,791,232unknown problem are in agreement with the analytical curve obtained by a Mie series solution.

minutes and the iterative solution is completed in 265 minutes. Some important details of the solution are as follows:

1) An MLFMA tree is constructed by including the geometry in a cubic box, which is recursively divided into sub-boxes (clusters). The tree structure has a total of 11 levels and the size of the smallest clusters is $192 \lambda \times$ $2^{-10}=0.1875 \lambda$. We note that 9 of these levels are active, i.e., MLFMA operations such as aggregations and translations are performed at 9 levels. Total number of clusters is 5,904,951 and the number of clusters in the lowest level, which includes the basis and testing functions, is 4,344,205.

2) The number of near-field interactions that are calculated directly is $3,732,101,432$. Calculation of these interactions dominates the setup time (177 minutes). Using a single-precision array, near-field interactions require total of $27.8 \mathrm{~GB}$ of memory, which is equally distributed among the processors using a load-balancing algorithm.

3) Truncation numbers in MLFMA for 2-digits of accuracy are $6\left(L_{\min }\right)$ and $546\left(L_{\max }\right)$ for the lowest-level and highest-level clusters, respectively. We sample the radiation and receiving patterns of the basis and testing functions at $\left(L_{\min }+1\right) \times\left(2 L_{\min }+2\right)$ points, which can be reduced to $\left(L_{\min } / 2+1\right) \times\left(2 L_{\min }+2\right)$ using the symmetry. These patterns are calculated analytically and stored in the memory before the iterations. CFIE requires the calculation of both radiation and receiving patterns, while the receiving patterns can be omitted in the EFIE solutions using a Galerkin scheme. Considering both $\theta$ and $\phi$ components of the patterns, total of $56 \mathrm{~GB}$ of memory is required in single-precision representation. These patterns are distributed among the processors considering the far-field partitioning of the matrix equation, which is usually different from the near-field partitioning for efficiency.

4) Due to the symmetry of the cubic clusters, the number of translation operators required to perform the cluster-tocluster interactions can be reduced significantly. Using a one-box-buffer scheme, the memory required to keep the translation operators is total of $2 \mathrm{~GB}$.

5) In a matrix-vector multiplication, radiated and incoming fields calculated during the aggregation and disaggregation processes, respectively, are kept in double-precision arrays requiring total of $79 \mathrm{~GB}$ of memory. To improve load-balancing, we employ different strategies to distribute the clusters in the lower and higher levels of the tree structure [3]. For the lower levels, each cluster is assigned to a single processor. A load-balancing algorithm is used to distribute the clusters in the lower levels equally among the processors. In the higher levels, each cluster is assigned to all processors partially by partitioning the radiation and receiving patterns. For the lower levels, some of the translations require communications between the processors while all the translations in the upper levels can be completed without any communication. For this problem with 33,791,232 unknowns, each matrix-vector multiplication is performed in 370 seconds.

To demonstrate the accuracy of the solutions, Fig. 1 presents the bistatic radar cross section (RCS) of the sphere from $160^{\circ}$ to $180^{\circ}$, where $180^{\circ}$ corresponds to the forward-scattering direction. We observe that the computational values that are sampled with $0.1^{\circ}$ intervals are very close to the analytical curve obtained by a Mie-series solution. The root-mean-square error of the RCS is only 0.547 decibels $(\mathrm{dB})$ in the $160^{\circ}-170^{\circ}$ range and $0.915 \mathrm{~dB}$ in the $170^{\circ}-180^{\circ}$ range.

\section{Solutions of Open Surfaces by EFIE}

For the solutions of the scattering and radiation problems involving open geometries such as depicted in Fig. 2, EFIE is inevitably used to formulate the problems since CFIE is not applicable to open surfaces. Unfortunately, EFIE usually produces ill-conditioned matrix equations that are difficult to solve iteratively [4]. Especially, as the problems size grows, strong preconditioners are required to obtain a quick convergence. On the other hand, it is also desirable to implement 


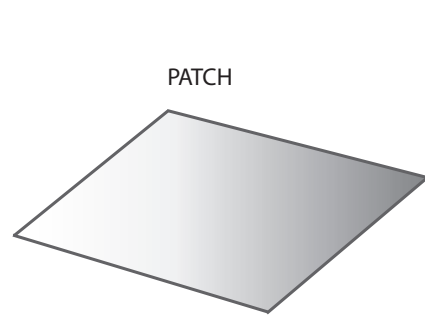

REFLECTOR ANTENNA

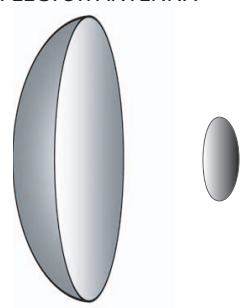

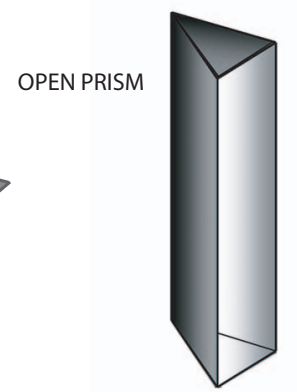

HALF SPHERE

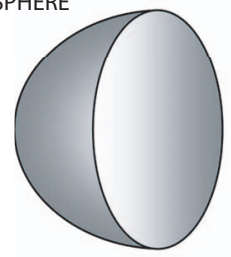

Fig. 2. Geometries involving open surfaces.

TABLE I

OPEN GEOMETRIES SOLVED BY MLFMA. THE "SIZE" COLUMN STANDS FOR THE MAXIMUM SIDE LENGTH IN TERMS OF $\lambda$.

\begin{tabular}{|c|c|c|c|c|}
\hline Problem & Freq. $(\mathrm{GHz})$ & Size $(\lambda)$ & Levels & Unknowns \\
\hline \hline P1 & 48 & 48 & 9 & 790,656 \\
\hline P2 & 96 & 96 & 10 & $3,164,544$ \\
\hline P3 & 192 & 192 & 11 & $12,662,016$ \\
\hline \hline HS1 & 9.25 & 18.5 & 8 & 159,452 \\
\hline HS2 & 18.5 & 37 & 9 & 638,392 \\
\hline HS3 & 37 & 74 & 10 & $2,554,736$ \\
\hline \hline OP1 & 20 & 35 & 9 & 182,780 \\
\hline OP2 & 40 & 69 & 10 & 731,896 \\
\hline OP3 & 80 & 139 & 11 & $2,929,136$ \\
\hline \hline RA & 3.73 & 92.6 & 10 & $2,515,103$ \\
\hline
\end{tabular}

efficient preconditioners with low complexities. In this manner, sparse approximate inverse (SAI) preconditioner is commonly used to accelerate the EFIE solutions [5]. However, even this type of robust preconditioners that are built from the nearfield interactions may become inefficient as the problem size grows. As a remedy, we propose AMLFMA, which is based on approximating the full matrix by systematically reducing the accuracy of MLFMA. By employing AMLFMA in an innerouter scheme, we develop an alternative robust preconditioner, which usually performs better than the SAI preconditioner.

AMLFMA is developed by carefully reducing the samples of the radiation and receiving patterns of the clusters in MLFMA. For this purpose, we use an approximation factor $a_{f}$, which is defined in the range from 0.0 to 1.0. Then, AMLFMA uses the reduced truncation numbers

$$
L_{l}^{r}=L_{\min }+a_{f}\left(L_{l}-L_{m i n}\right)
$$

for level $l$, where $L_{\min }$ is the truncation number defined for the lowest level and $L_{l}$ is the original truncation number (for level $l$ ) in the full MLFMA. When $a_{f}=1.0$, AMLFMA corresponds to the full MLFMA. On the other hand, as it decreases towards 0.0, AMLFMA becomes less accurate but
TABLE II

COMPARISON OF SAI AND AMLFMA PRECONDITIONERS FOR THE SOLUTIONS OF Open PRoblems.

\begin{tabular}{|c|cc|cc|}
\hline & \multicolumn{2}{|c|}{ SAI } & \multicolumn{2}{c|}{ AMLFMA } \\
\hline Problem & Iterations & Time (min.) & Iterations & Time (min.) \\
\hline P1 & 128 & 11 & 23 & 6 \\
\hline P2 & 195 & 95 & 36 & 46 \\
\hline P3 & 275 & 559 & 53 & 270 \\
\hline \hline HS1 & 174 & 5 & 24 & 3 \\
\hline HS2 & 321 & 37 & 44 & 18 \\
\hline HS3 & 547 & 289 & 70 & 111 \\
\hline \hline OP1 & 206 & 10 & 35 & 5 \\
\hline OP2 & 285 & 77 & 65 & 35 \\
\hline OP3 & 539 & 613 & 122 & 275 \\
\hline \hline RA & $>1000$ & - & 322 & 429 \\
\hline
\end{tabular}

much cheaper, especially if the number of levels is high. Our numerical experiments show that $a_{f}=0.2$ is a good choice.

To demonstrate the performance of the AMLFMA preconditioner, we consider various scattering problems that are depicted in Fig. 2 and also listed in Table I. One of these problems, namely, P3, is the largest ever reported EFIE problem. Each problem is solved by generalized minimal residual (GMRES) and flexible GMRES (FGMRES) methods, which use SAI and AMLFMA preconditioners, respectively. For the SAI preconditioner, near-field pattern is used to select the nonzero elements of the approximate inverse. In the FGMRES solutions, where AMLFMA is employed as the preconditioner, the inner solutions are performed by GMRES, which is further accelerated by SAI. The inner iterations are stopped when the residual error drops below 0.1 or the number of iterations reaches to 10 . Solutions of the scattering problems with $10^{-6}$ residual error are listed in Table II, where we observe a significant improvement by AMLFMA compared to SAI. All solutions are parallelized into 32 processes and performed on a cluster of quad-core Intel Xeon 5355 processors. For the problems that are solvable with SAI, solution times are reduced by about $50 \%$ using AMLFMA. For the reflector antenna problem denoted by RA, we observe that SAI cannot provide a convergence within 1000 iterations, while the same problem is solved by using AMLFMA in 322 iterations.

Finally, we present the results of the scattering problems involving metamaterial (MM) blocks constructed by the arrangement of the split-ring resonantors (SRRs) and thin wires (TWs). Since both SRRs and TWs are modelled by perfectly-conducting sheets, these scattering problems are formulated by EFIE. Fig. 3 depicts the power transmission for an SRR block, which is constructed by employing $4 \times 18 \times 11$ unit cells. The block is illuminated by a Hertzian dipole and the scattering problem is solved at $85 \mathrm{GHz}$ and $100 \mathrm{GHz}$. At $85 \mathrm{GHz}$, the SRR block is almost transparent and the power transmission is unity $(0 \mathrm{~dB})$ in the transmission region (left-hand side of the block). On the other hand, at $100 \mathrm{GHz}$, which is the resonance frequency of the SRRs [6], negative permeability is stimulated in the medium and we observe the shadowing effect. Using TWs in addition to SRRs, we construct composite MMs (CMMs), which show double-negative 


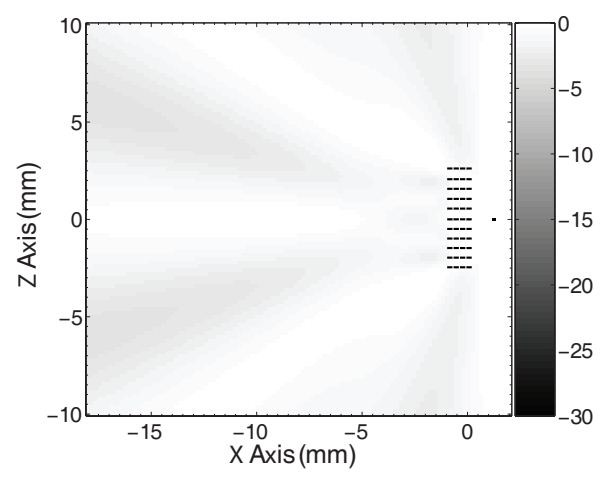

(a)

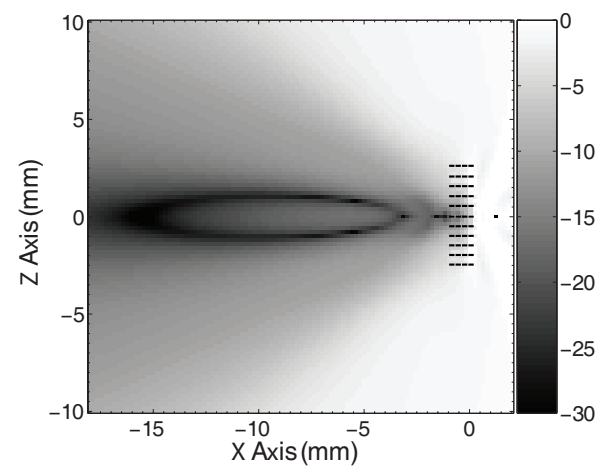

(b)

Fig. 3. Power transmission $(\mathrm{dB})$ for a $4 \times 18 \times 11$ SRR block when it is illuminated by a Hertzian dipole (shown by dot in the figure) at (a) $85 \mathrm{GHz}$ and (b) $100 \mathrm{GHz}$.

property around the resonance frequency. Fig. 4 depicts the power transmission for a CMM block, which is constructed by inserting TWs into $4 \times 18 \times 11$ SRR block. We observe that the CMM block prevents the power from passing into the transmission region at $85 \mathrm{GHz}$. However, the transmission through the block increases at $100 \mathrm{GHz}$, since both effective permittivity and permeability become negative.

For the solutions of the SRR and CMM problems, discretization of the geometries leads to 66,528 and 112,128 unknowns, respectively. Although these are relatively small problems compared to others considered in this paper, solutions of the MM problems are extremely difficult and need strong preconditioners. Because, MM structures present numerical resonances (in addition to the physical resonances) that inhibits a rapid convergence in the iterative solutions. As an example, using the SAI preconditioner, number of GMRES iterations for the SRR problem is 53 and 254 at $85 \mathrm{GHz}$ and $100 \mathrm{GHz}$, respectively, to reduce the residual error below $10^{-3}$. For much larger problems, SAI becomes insufficient so that we need AMLFMA for efficient solutions.

\section{CONCLUSIONS}

We report our efforts to solve large-scale problems in electromagnetics using preconditioned MLFMA. By developing robust implementations, we have been able to solve 12 million-unknown EFIE and 33-million-unknown CFIE problems. To our knowledge, these are the solutions of the largest

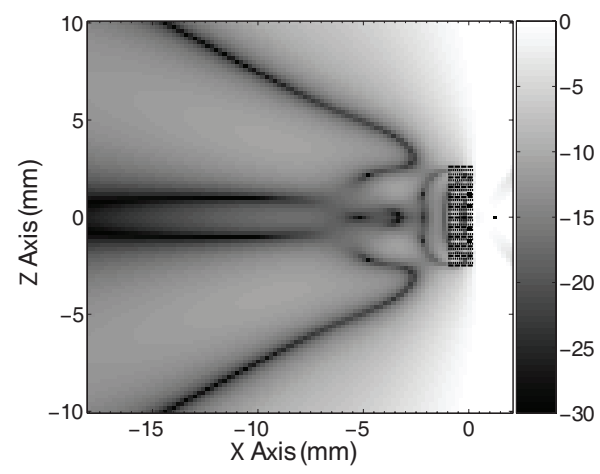

(a)

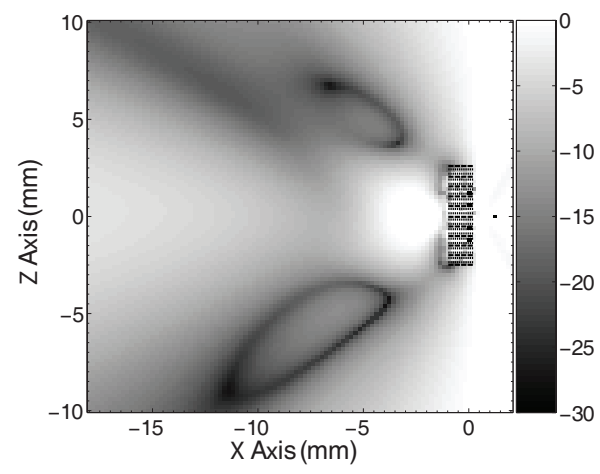

(b)

Fig. 4. Power transmission $(\mathrm{dB})$ for a CMM block constructed by thin wires and $4 \times 18 \times 11$ SRR block when it is illuminated by a Hertzian dipole (shown by dot in the figure) at (a) $85 \mathrm{GHz}$ and (b) $100 \mathrm{GHz}$.

integral-equation problems that have ever been reported. More examples, especially on large and complicated MM structures, will be provided during the presentation.

\section{ACKNOWLEDGMENT}

This work was supported by the Scientific and Technical Research Council of Turkey (TUBITAK) under Research Grant 105E172, by the Turkish Academy of Sciences in the framework of the Young Scientist Award Program (LG/TUBAGEBIP/2002-1-12), and by contracts from ASELSAN and SSM. Computer time was provided in part by a generous allocation from Intel Corporation.

\section{REFERENCES}

[1] J. Song, C.-C. Lu, and W. C. Chew, "Multilevel fast multipole algorithm for electromagnetic scattering by large complex objects," IEEE Trans. Antennas Propagat., vol. 45, no. 10, pp. 1488-1493, Oct. 1997.

[2] S. M. Rao, D. R. Wilton, and A. W. Glisson, "Electromagnetic scattering by surfaces of arbitrary shape," IEEE Trans. Antennas Propagat., vol. AP-30, no. 3, pp. 409-418, May 1982.

[3] Ö. Ergül and L. Gürel, "Efficient parallelization of multilevel fast multipole algorithm," in Proc. European Conference on Antennas and Propagation (EuCAP), 350094, 2006.

[4] L. Gürel and Ö. Ergül, "Comparisons of FMM implementations employing different formulations and iterative solvers," in Proc. IEEE Antennas and Propagation Soc. Int. Symp., vol. 1, 2003, pp. 19-22.

[5] B. Carpentieri, I. S. Duff, and L. Giraud, "Experiments with sparse preconditioning of dense problems from electromagnetic applications," CERFACS, Toulouse, France, Tech. Rep. TR/PA/00/04, 1999.

[6] L. Gürel, A. Ünal, and Ö. Ergül, "Electromagnetic modeling of splitring resonators," in Proc. 36th European Microwave Conference, 2006, pp. 303-305. 